\title{
CARTOGRAFÍA DEL PERMAFROST DE MONTAÑA EN LOS PIRINEOS ESPAÑOLES
}

\author{
ENRIQUe SERRANO ${ }^{1}$ \\ Carlos Morales ${ }^{2}$ \\ JuAn José GonZÁLEZ-TruebA ${ }^{3}$ \\ RAÚL MARTÍN ${ }^{4}$
}

\begin{abstract}
Resumen - En este trabajo se presenta un mapa de síntesis que ofrece una visión global de la distribución del permafrost en los Pirineos a escala regional. La elaboración del mapa está basada en la utilización de indicadores de permafrost (glaciares rocosos, gelifluxión, cavidades heladas, suelos ordenados, sondeos eléctricos verticales y registros térmicos de suelos) y un modelo digital del terreno (MDT) con información sobre la inclinación de las laderas y su orientación, la altitud y la radiación solar directa potencial. El mapa resultante contiene información sobre la distribución espacial del "permafrost probable" y del "permafrost posible", una aproximación que muestra el estado actual del conocimiento sobre permafrost en los Pirineos.
\end{abstract}

Palabras clave: Permafrost de montaña, cartografía, glaciares rocosos, SIG, Pirineos.

\begin{abstract}
Mountain Permafrost map of the Spanish Pyrenees. Since the 1980 's with the development of research on rock glaciers and periglacial landforms as permafrost indicators that the mountain permafrost is known to occur in the Pyrenees. In this paper we present a map of the distribution of mountain permafrost in the Pyrenees. The mapping is based on indicators of permafrost (rock glaciers, gelifluction processes, ice caves, patterned ground, vertical electric sounding and ground thermal regimes) and geographical information extracted from a Digital Elevation Model, such as slope angle and aspect, altitude and potential direct solar radiation. The map represents the spatial distribution of "probable" and "possible permafrost" and represents the present-day state of the art on Pyrenean permafrost.
\end{abstract}

Key words: Mountain permafrost, mapping, rock glaciers, GIS, Pyrenees.

Recebido: 14/11/2007. Revisto: 01/04/2009. Aceite: 01/06/2009.

1 Departamento de Geografía. Universidad de Valladolid. E-mail: serranoe@fyl.uva.es

2 Departamento de Geografía. Universidad de Valladolid. E-mail: cmorales@fyl.uva.es

3 Departamento de Geografía, Prehistoria y Arqueología. Universidad del País Vasco. E-mail: jjgtrueba@hotmail.com

4 Departamento de Ciencias. Universidad de San Luis (Campus, Madrid).

E-mail: martinra@madrid.slu.edu 
Resumo - CARTOGRAFIA DO PERMAFROST DE MONTANHA NOS PIRENÉUS ESPANHÓIS. Neste trabalho, apresenta-se um mapa que oferece uma visão global acerca da distribuição do permafrost de montanha nos Pirenéus espanhóis. A elaboração do mapa apoia-se na utilização de indicadores da presença de permafrost (glaciares rochosos, gelifluxão, grutas geladas, solos ordenados, sondagens eléctricas verticais e dados de temperatura do solo), bem como num modelo digital de terreno, do qual se derivaram mapas de declives, exposições e a radiação directa potencial. O mapa representa a distribuição do permafrost provável e do permafrost possível, numa cartografia que mostra o estado actual do conhecimento. Pirenéus.

Palavras-chave: Permafrost de montanha, cartografia, glaciares rochosos, SIG,

Résumé - CARTographie du PERMAFrost de MONTAgne DANS LES PyrÉNÉES ESPAGNOLES. On présente une carte de synthèse, offrant une vue d'ensemble de la répartition du permafrost dans les Pyrénées, à l'échelle régionale. La carte a été élaborée à partir d'indicateurs du permafrost (glaciers rocheux, gélifluxion, cavités gelées, sols ordonnés, sondages électriques verticaux et enregistrements thermiques dans les sols) et d'un modèle digital de terrain (MDT), prenant en compte l'inclinaison et l'orientation des versants, l'altitude et la radiation solaire directe potentielle. La carte résultante fournit des informations sur la répartition spatiale du «permafrost probable» et du «permafrost possible» et schématise l'état actuel des connaissances sur le permafrost dans les Pyrénées. Pyrénées.

Mots clés: Permafrost de montagne, cartographie, glaciers rocheux, SIG,

\section{INTRODUCCIÓN}

El permafrost, condición térmica del suelo o el sustrato donde las temperaturas bajo $0^{\circ} \mathrm{C}$ persisten al menos dos años consecutivos y posibilita la existencia de depósitos superficiales congelados, es un elemento fundamental de los sistemas morfogenéticos de la alta montaña. Su presencia está estrechamente relacionada en primer lugar con factores como la altitud y la topografía, pero también con los glaciares de montaña y su retroceso, el manto nival, los procesos geomorfológicos y la hidrología. El estudio del permafrost de montaña tiene un elevado interés para el conocimiento de la dinámica de la alta montaña y para la intervención en ella, ya sea para su conservación, la introducción de nuevos usos o para conocer su respuesta en el contexto del cambio global.

El estudio de la distribución del permafrost de montaña se inicia con la utilización de formas y procesos periglaciares como indicadores de su existencia: glaciares rocosos activos, derrubios afectados por flujo (protalus lobe), suelos ordenados o montículos de hielo (frost mounds), procesos de levantamiento por helada (frost heave), gelifluxión, y criorreptación. Todos ellos son útiles indicadores usados tanto en medios polares (Black, 1976; Harris, 1986; Clark, 1988; 
French, 2007), como en medios de montaña en latitudes templadas (Barsch, 1978; Washburn, 1979; Harris, 1982, 1988; Karte, 1983; Haeberli, 1985). Estas formas y procesos permiten una primera aproximación a su presencia y distribución, pero no su representación cartográfica. Haeberli propone el primer modelo empírico de distribución del permafrost a partir de las siete reglas de thumb, iniciándose en los Alpes la cartografía del permafrost mediante la elaboración de los modelos empíricos Permakart y Permamap (Keller, 1992; Hoetzle et al., 1993; Keller et al., 1998; Heginbottom, 2002). Ambos modelos diferencian entre permafrost probable y posible. La aplicación de estas metodologías permite aproximarse a la distribución del permafrost en medios de montaña mediante cartografías de detalle, a pequeña escala (King y Kalisch, 1998; Serrano et al., 2001; Tanarro et al., 2001, 2002), o a escala regional (Keller et al., 1998; King, 1996; Lieb, 1998; Lambiel y Reynard, 2001; Oberman y Mazhitoba, 2003; Heggen et al., 2005; Kellerer, 2005), y se han desarrollado diferentes modelos que integran variables topoclimáticas y biológicas (Permamod - Frauenfelder et al., 1998; Etzelmüller et al., 2001), nivales y con datos del terreno (Permebal - Stocker et al., 2002), o datos climáticos y térmicos del suelo (Permaclim - Guglielmin et al., 2003). Estudios regionales y análisis a gran escala de los Alpes señalan precisiones bajas (800 metros en Permakart - Keller et al., 1998), que denotan la ausencia de factores climáticos locales y regionales, geológicos o geomorfológicos. Según diferentes investigaciones el principal factor en la distribución del permafrost es la radiación solar directa (Tanarro et al., 2001, 2002; Kellerer-Pirklbauer, 2005; Julián y Chueca, 2007), en relación con la cobertura nival, o la altitud (Heggen et al., 2005); mientras para otros la temperatura media anual del aire no es indicadora de la temperatura del suelo (Oberman y Mazhitoba, 2003).

El uso de técnicas GIS permite una extrapolación eficaz a partir de un número más reducido de registros, que deben ser validados con mediciones puntuales sobre el terreno - registros térmicos continuos, medidas térmicas de la temperatura basal del manto nival (BTS), sondeos geoeléctricos, sondeos georadar y la realización de estimaciones y mapas a escalas regionales (Keller et al., 1998; Guglielmin y Sileto, 2000; Lambiel y Reynard, 2001; Lugon y Delaloyé, 2001; Oberman y Mazhitoba, 2003; Heggen et al., 2005; Kellerer, 2005; Janke, 2005; Fukui et al., 2007).

La representación cartográfica del permafrost puede realizarse por sus condiciones térmicas, dinámicas o espaciales. Los mapas basados en estados térmicos son muy útiles y permiten una aproximación a su estado - estable, metaestable, inestable, en equilibrio o desequilibrio ambiental (Harris, 1986; Oberman y Mazhitova, 2003), pero se requieren registros continuos y numerosos, pocas veces disponibles en la alta montaña. los mapas que representan el tipo de distribución espacial (permafrost continuo, discontinuo o esporádico) han sido abordados en estudios locales y regionales en montaña (Lambiel y Reynard, 2001; Serrano et al., 2001; Kellerer, 2005), pero ofrecen escasa precisión y son necesarios estudios detallados de muchos macizos para obtener resultados rigu- 
rosos. Desde los trabajos de Keller (1992), la precisión en la distribución del permafrost de montaña se limita a establecer la existencia "probable" de permafrost, cuando las condiciones favorecen su presencia y los indicadores son claros, o "posible", cuando existen condiciones propicias, pero no claras, y escasos indicadores morfogenéticos. Esta tipología es la más aceptada y utilizada en los mapas regionales sobre permafrost de montaña a partir de métodos cartográficos basados en observaciones de campo y modelos semiempíricos.

\section{OBJETIVOS Y ZONA DE ESTUDIO}

El objetivo de este trabajo es realizar el mapa del permafrost de los Pirineos españoles, como contribución al conocimiento del permafrost pirenaico. Se trata de una síntesis espacial del estado actual del conocimiento del permafrost de montaña, una aportación al conocimiento del dominio periglaciar y una herramienta útil para el análisis de la sensibilidad al cambio de la alta montaña pirenaica por causas naturales o antrópicas, así como para su utilización en la protección y conservación de los medios de montaña pirenaicos, buena parte de ellos catalogados como espacios naturales protegidos, y en los que el permafrost es un elemento significativo de su medio natural.

La alta montaña de los Pirineos se desarrolla por encima de los $2300 \mathrm{~m}$., con intensos procesos periglaciares activos por encima de $2400 \mathrm{~m}$. La posible existencia de permafrost ha sido señalada mediante indicadores geomorfológicos, suelos ordenados (Cailleux y Hupé, 1947; Monturiol, 1959; Hupé, 1962; Angely, 1967; Gómez Ortiz, 1987) y glaciares rocosos (Serrano y Agudo 1998, Serrano et al., 1999, 2006). Las investigaciones climáticas (Barrio et al., 1990; García Ruiz et al., 1990; Barrio, 1990) localizan el límite inferior del permafrost por encima de $2700 \mathrm{~m}$, y los estudios a partir de geoindicadores, registros térmicos y sondeos geofísicos (Serrano, 1996 1998; Lampre, 1994, 1998; Fabré et al., 1995; Serrano y Agudo, 1998; Serrano et al., 2001, 2002, 2006; Lugon et al., 2004; Chueca y Julián, 2004; Martín, 2006; Julián y Chueca, 2007) sitúan el permafrost continuo por encima de los 3000 -3 100 m s.n.m.

\section{METODOLOGÍA}

El mapa del permafrost se basa en la combinación e integración de datos de campo y fuentes publicadas en un modelo cartográfico digital mediante técnicas GIS. El mapa se ha elaborado con un número limitado de datos de campo directos obtenidos mediante diferentes técnicas. Los indicadores de permafrost utilizados para la elaboración del mapa son de dos tipos:

- Información térmica, en la que se han utilizado registros térmicos de la temperatura basal del manto nival (mediciones BTS) en los macizos de Posets, Maladeta y Telera (Serrano et al., 2001; Lugon et al., 2004; Martín, 2006; Julián 
y Chueca, 2007), registros continuos mediante datalogger (UTL-1), en los macizos de Monte Perdido y Posets (Serrano et al., 2001; Martín 2006), y la existencia de cavidades congeladas en el macizo de Monte Perdido (10 cavidades), complementados con la altitud de las isotermas de $0^{\circ} \mathrm{C},-2^{\circ} \mathrm{C}$ y $-6^{\circ} \mathrm{C}$.

- Indicadores geomorfológicos. Se han inventariado como indicadores de permafrost 14 glaciares rocosos activos (Serrano y Agudo, 1998; Serrano et al., 1999), 10 derrubios afectados por flujo (protalus lobe) (Serrano, 1998; Serrano y Agudo, 2001, 2002; Serrano et al., 2001), los montículos de ielo y suelos ordenados de Vallibierna, Maladeta, Posets y Monte Perdido (Boyé, 1952; Monturiol, 1959, Angely, 1967; Serrano et al., 2002; Serrano y Agudo 2004; García Ruiz y Martí Bono, 2004; Martín, 2006), así como la presencia de huellas de termokarst y heleros permanentes. Estos datos se han referenciado (coordenadas, altitud, orientación) y se han clasificado como indicadores de permafrost posible o probable.

Las técnicas de prospección y modelización cartográfica utilizadas siguen la línea establecida por Haeberli (1985) y la utilización de herramientas SIG:

- La información de los macizos utilizados como "áreas clave", donde existen estudios de detalle (Águalas-Infierno, Vignemale, Monte Perdido, Posets, Maladeta y Besiberri), se ha extrapolado al conjunto de la alta montaña pirenaica mediante el uso de técnicas SIG. Los indicadores de permafrost, las isotermas anuales de temperatura del aire, la altitud, la orientación y la pendiente han sido integradas para incorporar la información al mapa. A cada indicador se le han asignado unos caracteres de emplazamiento (altitud, orientación, pendiente, exposición) y se han clasificado como indicadores de permafrost posible o probable.

- Se ha elaborado un modelo TIN (Triangulated Irregular Network) a partir de un mapa vectorial con equidistancia de $100 \mathrm{~m}$ y Sistema de Proyección UTM (Confederación Hidrográfica del Ebro), y se ha construido un modelo digital de elevaciones (DEM) formado por una malla con celdas de 50x50m (4 849366 celdas). A cada celda se le ha asignado un valor de altitud, pendiente y orientación.

- La integración de la información aportada por los indicadores geomorfológicos periglaciares, la tasa de radiación solar y las temperaturas medias del aire con los datos derivados del modelo digital del terreno (pendientes, orientación, exposición) permiten elaborar el mapa final. Los valores de permafrost de cada celda se han determinado mediante el Map Calculator de los programas ArcView y ArcMap que utilizan álgebra de mapas, según criterios de altitud, pendiente, orientación, valores de la isoterma, tasas de radiación y altitud de indicadores de permafrost. De este modo, la información de los indicadores geomorfológicos, térmicos y de radiación solar incidente se extrapola a las celdas del mapa que poseen las mismas características, aplicando a cada píxel los valores 1 para el "permafrost posible", 2 para el "permafrost probable" y 0 cuando no hay permafrost (sin representación en el mapa). El área representada de permafrost posible y probable es un modelo raster en el que cada píxel tiene una superficie de $50 \times 50 \mathrm{~m}$. 
El mapa final (el original a E. 1:300 000) comprende $12123,415 \mathrm{~km}^{2}$ en proyección UTM-WGS (1984). La representación altimétrica combina un sombreado para realzar la hipsometría y la extensión del permafrost se representan mediante dos tonos de grises (mapa en anexo).

\section{RESULTADOS}

El mapa resultante contiene la distribución general del permafrost en los Pirineos españoles diferenciando 3 categorías fundamentales: 1) permafrost probable: donde la probabilidad de que exista permafrost es alta; 2) permafrost posible, donde existen condiciones puntuales para la presencia de permafrost, pero irregular o discontinuo; 3) sin permafrost.

El mapa muestra la existencia de permafrost en las zonas más altas de la alta montaña, con intromisiones en áreas más bajas a favor de las caras norte de los macizos más elevados. El permafrost posible se extiende desde el macizo de Bisaurín $\left(0^{\circ} 38^{\prime} \mathrm{W}\right)$ hasta la Tossa de Alp ( $\left.1^{\circ} 53^{\prime} \mathrm{E}\right)$, y por el sur hasta los $42^{\circ} 16^{\prime} \mathrm{N}$ de la Sierra del Cadí. La existencia a cotas bajas de permafrost posible en orientaciones septentrionales de las sierras con cumbres por encima de los $2500 \mathrm{~m}$ y paredes de gran desarrollo vertical (500-100 metros) (Telera, Tendeñera, Cotiella y Cadí), denota la importante influencia topoclimática en la presencia y distribución del permafrost posible. El permafrost probable queda limitado a las cumbres más altas de los principales macizos desde el Puig Pedrós $\left(1^{\circ} 47^{\prime} \mathrm{E}\right)$ hasta las cumbres de la Sierra de Collarada $\left(0^{\circ} 29^{\prime} \mathrm{W}, 42^{\circ} 41^{\prime} \mathrm{N}\right)$ y el macizo de Balaitús $\left(0^{\circ} 20^{\prime} \mathrm{W}, 42^{\circ} 53^{\prime} \mathrm{N}\right)$ al norte. Son los macizos más elevados y con mayor extensión por encima de los $2700 \mathrm{~m}$, donde la importancia del permafrost probable es mayor. Argualas-Infierno, Panticosa, Vignemale, Monte Perdido, La Munia, Punta Suelza, Bachimala, Perdiguero, Posets, Maladeta, Besiberri, Colomers, Peguera, Estats, Tossa Plana de Lles y Puig Pedrós, muestran la existencia de un permafrost probable que no se ajusta exclusivamente a las condiciones de exposición.

\section{CONCLUSIONES}

El uso de indicadores geomorfológicos y datos térmicos de las áreas clave integrados con la orientación, altitud y pendientes mediante un SIG en un modelo simple, que permite conocer la distribución posible y probable del permafrost de montaña en la vertiente española de los Pirineos a una escala media. Esta se concreta en la existencia de permafrost posible por encima de los $2400 \mathrm{~m}$ en las orientaciones norte, que desciende hasta los $2000 \mathrm{~m}$ al pie de grandes paredes a favor de las condiciones topoclimáticas, y asciende a $2650 \mathrm{~m}$ en las meridionales. A estas altitudes pueden existir condiciones de permafrost que generan un permafrost esporádico. El permafrost probable se presenta en las 
orientaciones septentrionales a partir de los $2700 \mathrm{~m}$, con presencia en caras norte hasta los $2630 \mathrm{~m}$, mientras que en las orientaciones meridionales se sitúa por encima de los $2800 \mathrm{~m}$. Se corresponde con un permafrost discontinuo a continuo, con una intensa influencia topoclimática, limitado por las temperaturas medias anuales del aire y propiciado sólo en orientaciones favorables. Para un conocimiento más detallado de los límites altitudinales y la dinámica del permafrost, es necesario validar esta cartografía con análisis de detalle y observaciones minuciosas en los macizos que se ha detectado la presencia potencial de permafrost posible y probable pero no existe información.

El mapa del permafrost es una primera aproximación útil para la consideración de los espacios con hielo, junto a los glaciares pirenaicos, en la gestión de la alta montaña y en la conservación de espacios naturales, que en su mayor parte coinciden con Espacios Naturales Protegidos (Nacionales y Autonómicos). El conocimiento de su distribución posible y probable es una ayuda a la gestión y al conocimiento de la estructura y dinámica de ecosistemas y hábitats de la alta montaña pirenaica en un contexto de cambio global.

\section{AGRADECIMIENTOS}

Este trabajo ha sido financiado por el Ministerio de Ciencia y Tecnología, proyecto CGL2007-65295, y los Fondos Europeos de Desarrollo Regional (FEDER). Agradecemos las sugerencias de los revisores anónimos.

\section{BIBLIOGRAFÍA}

Angely A G (1967) Anciens glaciers dans l'est des Pyrénées centrales. Revue Géographique des Pyrénées et du Sud-Ouest, 38: 5-28.

Barrio G del (1990) El régimen térmico estacional en alta montaña. In García Ruiz J M (ed.). Geoecología de las áreas de montaña. Geoforma ediciones, Logroño: 115-144.

Barrio G del, Creus J, Puigdefábregas J (1990) Thermal seasonality on the high mountain belts of the Pyrenees. Mountain Research and Development, 10 (3): 227-233.

Barsch D (1978) Active rock glaciers as indicators for discontinuous alpine permafrost. An example from the Swiss Alps. Proceeding Third International Conference on Permafrost, Edmonton, vol. 1: 349-352.

Black R F (1976) Features indicatives of permafrost. Annual Review of Earth and Planetary Sciences, 4: 75-94.

Boyé M (1952) Gélivation et cryoturbation dans le Massif du Mont Perdú (Pyrénées centrales). Pirineos, 23: 5-30.

Chueca J, Julián A (2004) Determinación de la existencia de permafrost en la Sierra de Telera (Pirineo Central español) a partir de sondeos verticales y mediciones BTS. Boletín de la Real Sociedad Española de Historia Natural, 99: 65-74.

Clark M J (1988) Advances in periglacial geomorphology. Wiley \& Son. Chichester. 
Etzelmüller B, Odegard R S, Berthling I, Sollid J L (2001) Terrain parameters and remote sensing data in the analysis of permafrost distribution and periglacial processes: principles and examples from Southern Norway. Permafrost and Periglacial Processes, 12: 79-92.

Fabré D, García F, Evin M, Martínez R, Serrano E, Assier A, Smiraglia C (1995) Structure interne du glacier rocheux actif de las Argualas (Pyrenées Aragonaises, Espagne). La Houille Blanche, 5-6: 144-147.

Frauenfelder R, Allgower B, Haeberli W, Hoelzle M (1998) Permafrost investigations with GIS - a case of study in the Fletschhorn area, Wallis, Swiss Alps. Proceeding of the $7^{\text {th }}$ International Conference on Permafrost, Yellowknife: 551-556.

French H M (2007) The periglacial environment. Wiley and Son, Chichester.

Fukui K, Fujii Y, Mikhailov N, Ostanin O, Iwahana G (2007) The lower limit of mountain permafrost in the Russian Altai Mountains. Permafrost and Periglacial Proceses, 18: 129-136.

García Ruiz J M, Martí Bono C E (2001) Mapa geomorfológico del Parque Nacional de Ordesa y Monte Perdido. O.A.P.N., $\mathrm{M}^{\mathrm{o}}$ de Medio Ambiente, Madrid.

García Ruiz J M, Alvera B, Barrio G Del, Puigdefábregas J (1990) Geomorphic processes above the timberline in the Spanish Pyrenees. Mountain Research and Development, 10(3): 201-214.

Gómez Ortíz A (1987) Contribució geomorfológica a l'estudi dels espais supraforestals pirenencs. Genesi, organització $i$ dinámica dels modelats glacials i periglacials de la Cerdanya i Alt Urgell. Institut Cartográfic de Catalunya, Barcelona.

Guglielmin M, Siletto G B (2000) Carta della Criosfera. SELCA, Lombardía.

Guglielmin M, Aldighieri B, Testa B (2003) PERMACLIM: a model for distribution of mountain permafrost, based on climatic observations. Geomorphology, 51 (4): 38-47.

Haeberli W (2000) Modern research perspectives relating to permafrost creep and rock glaciers: a discussion. Permafrost and Periglacial Processes, 11: 290-293.

Haeberli W (1985) Creep of mountain permafrost: internal structure and flow of alpine rock glaciers. Mitteilungen der Versuchsanstalt für Wasserbau, Hydrology und Glaziology, 77, Zurich.

Harris S A (1986) The permafrost environments. Croom Helm, Londres.

Harris S A (1982) Identification of permafrost zones using selected periglacial landforms. Proceeding of the Four Canadian Permafrost Conference: 49-58.

Harris S A (1988) The alpine periglacial zone. In Clark M J (ed.) Advances in Periglacial geomorphology. Wiley and Son, New York: 145-189.

Heginbottom J A (2002) Permafrost mapping: a review. Progress in Physical Geography, 26: 623-642.

Hupé P (1961) A propos des sols polygonaux et striés des Pyrénées. Compte-rendu Som. Seacn. Societé Geologique de France, 8: 228-229.

Janke J R (2005) The occurrence of alpine permafrost in the Frost Range of Colorado. Geomophology, 67: 375-389.

Karte J (1983) Periglacial phenomena and their significance as climatic and edaphic indicators. GeoJournal, 74: 329-340

Keller F (1992) Automated mapping of mountain permafrost using the program PERMAKART within the Geographical System ARC/INFO. Permafrost and Periglacial Processes, 3: 133-138.

Keller F, Frauenfelder R, Gardaz J M, Hoezle M, Kneisel C, Lugon R, Phillips M, Reynard E, Wenker L (1998) Permafrost map of Switzerland. In Lewkowictz A G, Allard M (eds.) Proceeding $7^{\circ}$ International Permafrost Conference, Université de Laval, Yellowknife: 557-562 . 
Kellerer-Pirklbauer A (2005) Alpine permafrost occurrence and its spatial limits: first results from the eastern margin of the European Alps. Norwegian Journal of Geography, 59: 184-193.

King L (1986) Zonation and ecology of high mountain permafrost in Scandinavia. Geografiska Annaler, 68 (A-3):131-139.

King L, Kalisch A (1998) Permafrost distribution and implications for construction in the Zermatt area, Swiss Alps. In Lewkowictz A G, Allard M (eds.) Proceeding $7^{\text {th }}$ International Permafrost Conference, Université de Laval, Yellowknife: 569-574.

Lambiel C, Reynard E (2001) Regional modelling of present, past and future potential distribution of discontinuous permafrost based on rock glacier inventory in the Bagnes-Héémence area (western Swiss Alps). Norwegian Journal of Geography, 55: 219-223.

Lampre F (1998) Estudio Geomorfológico de Ballibierna (macizo de la Maladeta, Pirineo aragonés): modelado glacial y periglacial. Consejo de protección de la naturaleza de Aragón, Zaragoza.

Lampre F (1994) La línea de equilibrio glaciar y los suelos helados en el Macizo de la Maladeta (Pirineo aragonés): evolución desde la Pequeña Edad de Hielo y situación actual. In Martí Bono C, García Ruíz J M (eds.) El glaciarismo surpirenaico: nuevas aportaciones. Geoforma Ediciones, Logroño: 125-142.

Lieb G K (1998) High mountain permafrost in the Austrian Alp (Europe). In Lewkowictz A G, Allard M (eds.) Proceeding $7^{\text {th }}$ International Permafrost Conference. Université de Laval, Yellowknife: 663-668.

Lugon R, Delaloyé R (2001) Modelling Alpine permafrost distribution, Val de Réchy, Valais Alps (Swizerland). Norwegian Journal of Geography, 55: 224-229.

Lugon R, Delaloyé R, Serrano E, Reynard E, Lambiel C, González Trueba J J (2004) Permafrost and Little Ice Age relationships, Posets massif, Central Pyrenees, Spain. Permafrost and Periglacial Processes 15: 207-220.

Martín R (2006) Estudio comparativo de formas y procesos glaciares y periglaciares desde la Pequeña Edad del Hielo: Altas Latitudes noruegas (Spitsbergen y Jotunheimen) y Altas Altitudes españolas (Pirineos, Sistema Central y Teide). Tesis Doctoral. Universidad Autónoma de Madrid. Madrid.

Monturiol J (1959) Sobre una forma periglaciar descubierta en el macizo de Vallibierna (Pirineo central). Notas y comunicaciones del I.G.M.E., 55: 59-70.

Oberman N G, Mazhitova G G (2003) Permafrost mapping of northeast European Russian based on the period of climatic warming 1970-1995. Norwegian Journal of Geography, 57:111-120.

Serrano E (1998) Geomorfología del Alto Gallego (Pirineo aragonés). Fundación Fernando El Católico, Zaragoza.

Serrano E (1996) Líneas de equilibrio glaciar, glaciares rocosos y paleoambiente postglaciar en la alta montaña pirenaica (macizo de Panticosa, Pirineo aragonés). In Pérez Alberti A, Martini P, Chesworth W, Ramil-Rego P (eds.) Dinámica y evolución de medios cuaternarios. Xunta de Galicia, Santiago de Compostela: 157-170.

Serrano E, Agudo C (1998) Glaciares rocosos activos de los Pirineos. Implicaciones ambientales. In Gómez Ortiz (eds.) Procesos biofísicos actuales en medios fríos. Universidad de Barcelona, Barcelona: 133-154.

Serrano E, Agudo C, Martínez de Pisón E (1999) Rock glaciers in the Pyrenees. Permafrost and Periglacial Processes, 10: 101-106.

Serrano E, Agudo C, Delaloyé R, González Trueba J J (2001) Permafrost distribution in the Posets massif, Central Pyrenees. Norwegian Journal of Geography 55: 245-252. 
Serrano E, Agudo C, González Trueba J J (2002) El dominio de alta montaña periglaciar en el macizo del Posets. In Serrano E, García A (eds.) Periglaciarismo en montaña y altas latitudes. Universidad de Valladolid, Valladolid: 163-184.

Serrano E, Agudo C, González Trueba J J (2002) La deglaciación de la alta montaña del macizo del Posets (Pirineo aragonés). Morfología, evolución y fases morfogenéticas glaciares. Cuaternario \& Geomorfología, 16: 11-126.

Serrano E, Martínez de Pisón E, Agudo C (2001) El medio periglaciar de alta montaña en el Pirineo Central: aportaciones recientes. In Peña J L, Sanchez M, Lozano M V (eds.) Procesos y formas periglaciares en la montaña mediterránea. Instituto de Estudios Turolenses, Teruel: 45-62.

Serrano E, San José J J, Agudo C (2006) Rock glacier dynamics in a marginal periglacial high mountain environment: flow, movement (1991-2000) and structure of the Argualas rock glacier (Pyrenees). Geomorphology, 74: 285-296.

Stocker M, Hoelzle M, Haeberli W (2002) Modelling alpine permafrost distribution based on energybalance data: a first step. Permafrost and Periglacial Processes, 13: 271-282.

Tanarro I, Hoelzle M, García A, Ramos M, Gruber S, Gómez A, Piquer M, Palacios D (2002) Elaboración de un modelo para calcular la distribución del permafrost en el Corral del Veleta (Sierra Nevada) mediante la utilización de SIG. In Serrano E, García A (eds.) Periglaciarismo en montaña y altas Latitudes. Universidad de Valladolid, Valladolid: 125-144.

Tanarro I, Hoelzle M, García A, Ramos M, Gruber S, Gómez A, Piquer M, Palacios D (2001) Permafrost distribution modelling in the mountains of Mediterranean: Corral del Veleta, Sierra Nevada, Spain. Norwegian Journal of Geography, 55: 253-260.

Washburn A L (1979) Geocryology. A survey of periglacial processes and enviroments. Arnold, London. 
PERMAFROST DE MONTAÑA EN LOS PIRINEOS ESPAÑOLES MOUNTAIN PERMAFROST IN THE SPANISH PYRENEES

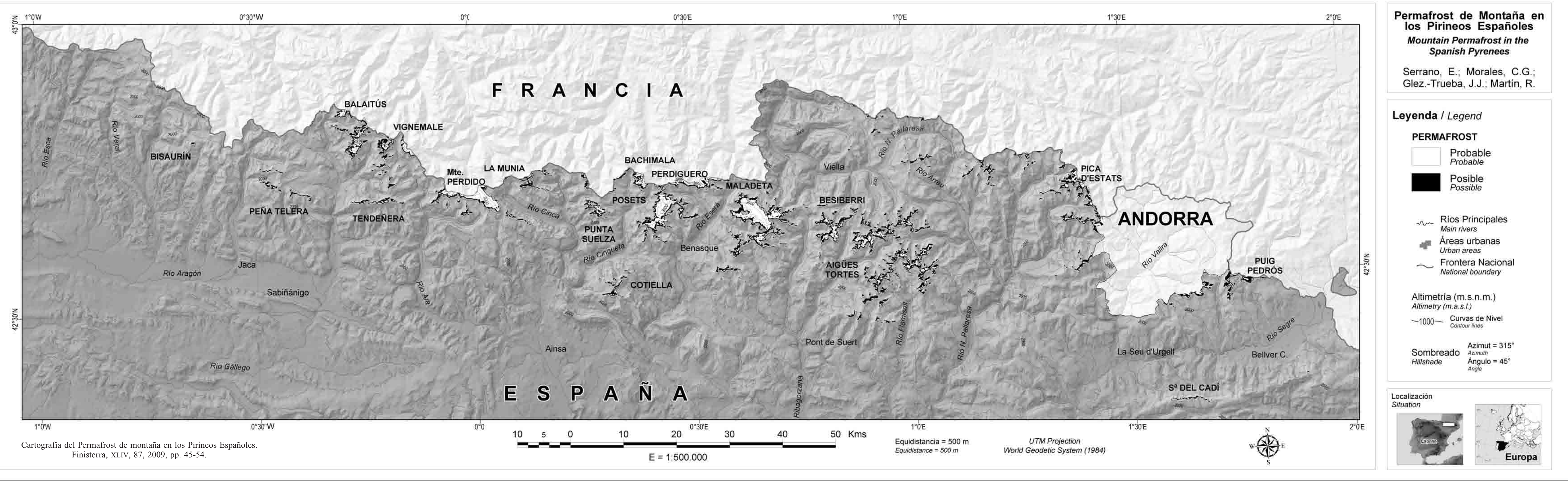

